\title{
Influence of Pilot Signal on Directivity of Self-Phased Arrays Under Conditions of Multipath Propagation
}

\author{
Sergey L. Loyka, Member, IEEE
}

\begin{abstract}
This paper presents an investigation of a pilot signal influence on directivity of self-phased array under conditions of multipath propagation (either reflections or jamming signals). A suggestion is made that an average reduction in directivity owing to the cutting off (or "dropping out") of some channels of the array be used as a criterion of this influence. It has been shown that an essential reduction in directivity is possible in the case of weak pilot signal or large (close to unity) reflection coefficient. If the level of direct pilot signal is higher than the cutoff threshold, the average reduction in directivity does not exceed $3 \mathrm{~dB}$, although substantially greater reduction (presence of "ejections") can be observed in certain specific cases. A reduction in directivity due to reflections can be removed by means of enhancing the sensitivity of the pilot signal channel. The method suggested in this paper can also be used in order to estimate influence of several reflections on self-phased array directivity.
\end{abstract}

Index Terms - Directivity, multipath propagation, self-phased array.

\section{INTRODUCTION}

$\mathbf{S}$ ELF-PHASED arrays have lately received wide application in telecommunications systems. They have a number of advantages in comparison with conventional antenna arrays. However, the electromagnetic environment can essentially influence their operation. Presence of reflections (multipath propagation) or jamming (interference) signals at the pilot signal frequency can lead to significant deterioration of a self-phased array operation.

The self-phased array operation under conditions of multipath propagation was studied in [1] and [2]. In particular, dependence of directivity of such an array on reflected signal parameters has been investigated. However, the influence of the total (direct plus reflected) pilot signal amplitude on directivity was not taken into account. As detailed investigations show, a reflected pilot signal can significantly influence performance of a self-phased array under conditions of large reflections and/or a weak pilot signal. We will consider this problem in more detail using the receiving self-phased array structure given in [1] (see Fig. 1). In this structure, each selfphasing unit consists of two filters tuned to the frequency of the primary (information) and pilot signals, a limiter, and a mixer (see Fig. 2). The primary and pilot signals are passed to the mixer input. A signal at intermediate frequency, which is

Manuscript received October 16, 1996; revised November 20, 1997. This paper was supported in part by the Soros Foundation.

The author is with the Electromagnetic Compatibility Laboratory, Belorussian State University of Informatics and Radioelectronics, Minsk, 220027 Republic of Belarus.

Publisher Item Identifier S 0018-9375(98)01716-5.

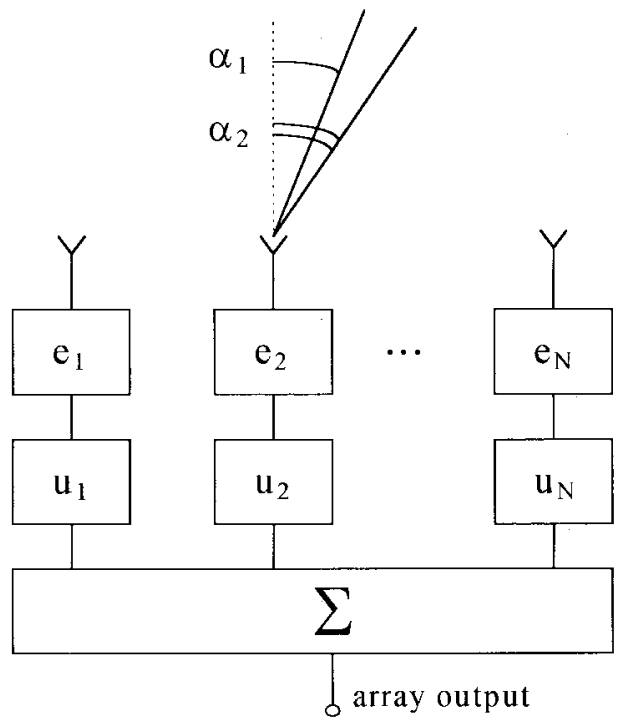

Fig. 1. Self-phased linear array structure; $e_{1}-e_{N}$-array elements; $u_{1}-u_{N}$-self-phasing units; $\Sigma$-adder.

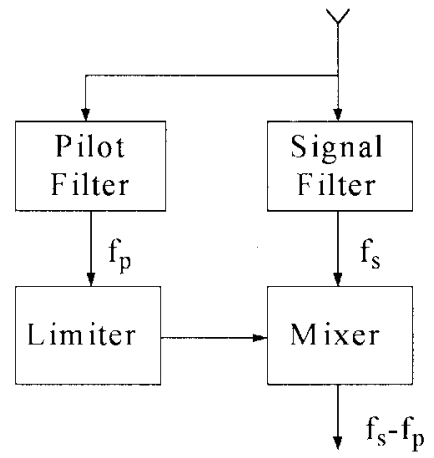

Fig. 2. Self-phasing unit structure; $f_{s}, f_{p}$-primary signal and, consequently, pilot frequencies.

equal to the difference of primary and pilot signal frequencies, is present at the mixer output.

Since the pilot signal is subjected to a limiting process prior to the mixer, we can assume that the channel of the pilot signal has threshold performance: if the amplitude of the pilot signal at the input of a self-phasing unit exceeds the threshold value (the receive sensitivity) $A_{\min }$ then the channel operates properly (provided that the amplitude of the primary signal exceeds the sensitivity level); if the amplitude of the pilot signal is lower than the threshold level then the channel is cut off (the "drop out" occurs). In fact, $A_{\min }$ is the cutoff threshold of the channel at the input of the self-phasing unit. Cutting off 
some channels will reduce the level of the primary signal at the array output and, consequently, decrease the directivity.

In this paper, we will consider influence of the total (direct + reflected) pilot signal on directivity of a receiving self-phased array. It will be shown how directivity of the self-phased array depends on the reflection parameters and on the level of the direct pilot signal.

\section{The Analysis of Self-Phased Array DiRECTIVITY FOR ONE REFLECTED Signal}

In the case of the threshold amplitude characteristic of the pilot-signal channel, directivity of self-phased array under conditions of multipath propagation can be written as follows (for detailed discussion, see Section IV):

$$
G=G_{p} \cdot G_{s} \cdot G_{0}
$$

where $G_{0}$ is the array directivity in the absence of reflections ( $G_{0} \approx N$ when directivity of an array element is close to unity; further, we will consider just this specific case: $N$ is the number of array elements); $G_{p}$ is reduction in directivity due to cutting off some channels (influence of reflections by way of the pilot signal channel); and $G_{s}$ is reduction in directivity owing to variations in amplitude, and phase of the primary signal (influence of reflections by way of the primary signal channel) [1]. The last parameter was comprehensively investigated in [1], [2] for the case of one reflected signal. Further, we will consider the parameter $G_{p}$ which can be defined as

$$
G_{p}=1-\frac{N_{d}}{N}
$$

where $N_{d}$ is the number of the cutoff channels. This equation can be justified as follows (now we do not take into account $G_{s}$ ). If there are $N_{d}$ cutoff channels, then $G=N-N_{d}=$ $N\left(1-N_{d} / N\right)=G_{0} G_{p}$, where $G_{p}$ is given by (2).

Let us now consider one channel of the array. For the case of one reflected signal, the condition for a proper channel operation looks like

$$
J=\frac{A_{\text {total }}}{A_{\text {min }}}=\beta \cdot \sqrt{1+a^{2}+2 \cdot a \cdot \cos \theta} \geq 1
$$

where $A_{\text {total }}$ is the amplitude of the total pilot signal at the input of the self-phasing unit, $\beta=A / A_{\min }$ is a relative level of the direct pilot signal, $A$ is amplitude of the direct pilot signal, $\theta$ is phase shift between the direct and reflected pilot signal at the input of the array element, $a=A_{r} / A$ is reflection coefficient, and $A_{r}$ is amplitude of the reflected pilot signal. Here we assume that array elements are omnidirectional (the directional properties of elements can be easily taken into account if necessary) and that all channels have identical parameters.

The condition for cutting off the channel is $J<1$. Analysis of the last expression and (3) reveals a peculiar "double threshold effect." The essence of this effect is explained in the following. The maximum suppression of the pilot signal (the worst case) occurs when $\theta=\pi$ and $J=\beta(1-a)$. The first threshold value $\beta_{m 1}$ is obtained from the following condition:

$$
\beta_{m 1} \cdot(1-a)=1 \Rightarrow \beta_{m 1}=\frac{1}{1-a}
$$

(for the case of $0 \leq a<1$ ). If $\beta \geq \beta_{m 1}$, then all channels will operate properly and $G_{p}=1$. In this case, the presence of reflection does not result in the deterioration of array performance owing to the pilot signal influence. The maximum amplification of the pilot signal is achieved when $\theta=2 \pi k$ ( $k$-integer) and $J=\beta(1+a)$. The second threshold value $\beta_{m 2}$ is derived from the following condition:

$$
\beta_{m 2} \cdot(1+a)=1 \Rightarrow \beta_{m 2}=\frac{1}{1+a} .
$$

If $\beta<\beta_{m 2}$, then all channels of the array will be cut off and $G_{p}=0$. If $\beta_{m 2} \leq \beta \leq \beta_{m 1}$, then $0 \leq G_{p} \leq 1$. The specific magnitude of $G_{p}$ is determined by array geometry and the value of $\theta$ for each channel. Thus, the threshold values $\beta_{m 1}$ and $\beta_{m 2}$ divide the whole range of $\beta$ into three subranges: subrange $1-\left[0, \beta_{m 2}\right]$; subrange $2-\left[\beta_{m 2}, \beta_{m 1}\right]$; and subrange 3 - $\left[\beta_{m 1}, \infty\right]$. For subrange 3 , reflection has no effect on directivity; for subrange 1, directivity is equal to zero (the array is fully cut off), and for subrange 2 , the effect takes intermediate values.

Similarly, using (4) and (5), one can define two threshold levels for $a$

$$
\begin{array}{ll}
a_{m 1}=\frac{\beta-1}{\beta}, & \beta \geq 1 \\
a_{m 2}=\frac{1-\beta}{\beta}, & \beta<1 .
\end{array}
$$

If $a<a_{m 1}$ then all channels will operate properly and $G_{p}=1$. If $a<a_{m 2}$ then all channels will be cut off and $G_{p}=0$. If $a_{m 2} \leq a$ or $a_{m 1} \leq a$, then $0 \leq G_{p} \leq 1$.

As (4) and (6) show, presence of the reflected signal can lead to a change in the array directivity in the case of a weak pilot signal and/or large (close to unity) reflection coefficient.

Let us now consider the case when $\beta_{m 2} \leq \beta \leq \beta_{m 1}$ and determine the threshold value $\theta^{*}$ with the use of the following:

$$
\beta \cdot \sqrt{1+a^{2}+2 \cdot a \cdot \cos \theta^{*}}=1, \quad \beta \in\left[\beta_{m 2}, \beta_{m 1}\right] .
$$

Using this equation, we obtain the expression for $\theta^{*}$ :

$$
\theta^{*}=\arccos \left(\frac{1-\beta^{2}\left(1+a^{2}\right)}{2 \cdot a \cdot \beta^{2}}\right) .
$$

If $-\theta^{*} \leq \theta \leq \theta^{*}$, then the channel will operate properly. In this case, inequality (3) is fulfilled. If $\theta^{*}<\theta \leq \pi$ or $-\pi \leq \theta<-\theta^{*}$, then inequality $J<1$ is fulfilled and the channel will be cut off. We should note that (8) and (9) are inapplicable when $a=0$. In this case, $\theta^{*}=\pi$. Expressions for the threshold levels $\beta_{m 1}$ and $\beta_{m 2}$ can also be obtained from (9) and from the restriction for the argument of the arccos function.

When the path difference between the direct and reflected signals or the phase shift during reflection is random, the reduction in directivity is random too. Let us calculate the average reduction in directivity using the following method. First, the probability of cutting off one channel is calculated. Then we calculate the average number of cutoff channels and, using (2) - the average reduction in directivity. This method is valid when $N>10$ (see Fig. 5 and the discussion below). 
We assume that $\theta$ has uniform distribution at the interval $[-\pi, \pi]$ (this assumption is valid for many practical cases, namely when the path difference between the direct and the reflected signal is comparable to or greater than the wavelength). The probability density function takes the following form:

$$
\omega(\theta)=\frac{1}{2 \pi}
$$

and the probability of cutting off a channel can be written as follows:

$$
P_{d}=1-\int_{-\theta^{*}}^{\theta^{*}} \omega(\theta) d \theta=1-\frac{\theta^{*}}{\pi} .
$$

Further, we can use this probability and obtain an expression for the average number of cutoff channels

$$
\bar{N}_{d}=N \cdot P_{d}=N \cdot\left(1-\frac{\theta^{*}}{\pi}\right) .
$$

Using (2) and (9), we obtain the formula for an average reduction in the array directivity due to cutting off channels

$$
\bar{G}_{p}=\frac{1}{\pi} \cdot \arccos \left(\frac{1-\beta^{2}\left(1+a^{2}\right)}{2 \cdot a \cdot \beta^{2}}\right) .
$$

The dependence $\bar{G}_{p}(a, \beta)$ is given in Fig. 3. This figure shows that the curve $\bar{G}_{p}(a, 1)$ divides the whole set of dependencies $\bar{G}_{p}(a, \beta)$ into two incoherent subsets: subset $1-\beta>1$ and subset $2-\beta<1$. For subset 1 , there is a minimum in the dependence $\bar{G}_{p}(a, \beta)$. The position of this minimum can be found using (13)

$$
a_{\min }=\sqrt{1-\frac{1}{\beta^{2}}} .
$$

Accordingly, we obtain expression for a minimum of $\bar{G}_{p}$

$$
\bar{G}_{p, \min }=\frac{1}{\pi} \cdot \arccos \left(-\frac{\sqrt{\beta^{2}-1}}{\beta}\right) .
$$

Equation (15) determines the greatest average reduction in directivity possible. For $\beta \geq 1$, the average reduction in directivity does not exceed $3 \mathrm{~dB}$. A significant reduction in directivity is possible for a low-level pilot signal (a small value of $\beta$ ) and for a large reflection coefficient. If the level of the pilot signal is lower than the threshold level, presence of reflections leads to an increase of average directivity in comparison with the case without reflections when directivity is equal to zero.

Equations (4) and (13) can be used for array design as follows. If there is a reflection and the reflection coefficient $a$ is known, then it is necessary to decrease the cutoff threshold (the receive sensitivity level) of the pilot signal channel in order to prevent cutting off the channels and, accordingly, reducing the directivity. The sensitivity level is to be decreased to

$$
A_{\min }^{*}=A_{\min } \cdot(1-a) .
$$

In this case, all units will operate properly and, accordingly, reduction in the directivity will not occur (provided that the array operates properly in the absence of reflection). If the permissible average reduction in directivity $\bar{G}_{p}$ and reflection

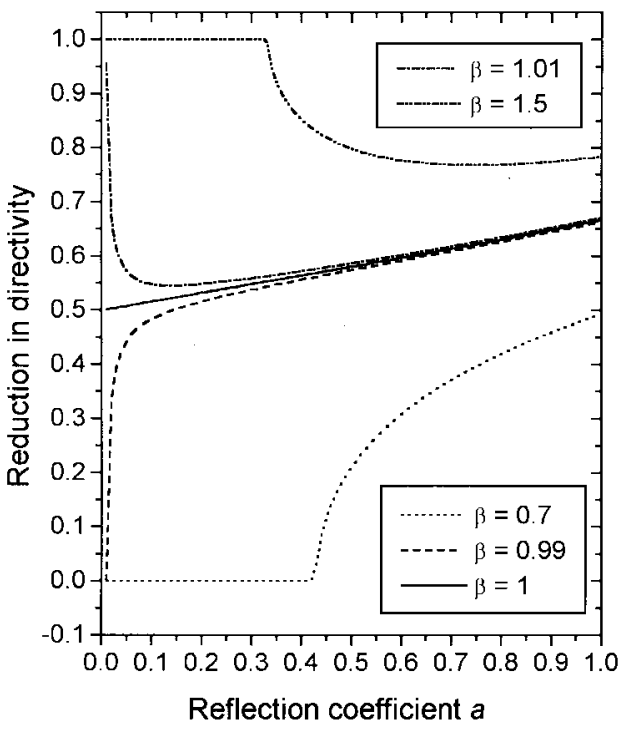

Fig. 3. Reduction in directivity against reflection coefficient (13).

coefficient $a$ are specified, then the required decrease (or increase) in sensitivity level can be calculated with the use of (13)

$$
A_{\min }^{*}=A_{\min } \cdot \sqrt{1+a^{2}+2 \cdot a \cdot \cos \left(\pi \bar{G}_{p}\right)} .
$$

It should be noted that for $\bar{G}_{p}=1$, (17) transforms to (16). For a 3-dB permissible average reduction in directivity $\left(\bar{G}_{p}=1 / 2\right)$, one obtains

$$
A_{\min }^{*}=A_{\min } \cdot \sqrt{1+a^{2}}
$$

and for $a=1$, a permissible increase of sensitivity level is equal to $3 \mathrm{~dB}$. It is physically explained by the fact that combining of the direct and reflected rays in phase leads to an increase of the pilot signal amplitude at the array input and, consequently, the sensitivity level of the pilot signal channel can be increased.

The dependence $A_{\min }^{*} / A_{\min }$ on $a$ for various values of $\bar{G}_{p}$ is represented in Fig. 4. This figure shows that the required decrease (or increase) of sensitivity level significantly depends on $\bar{G}_{p}$. For $\bar{G}_{p}=-0.5 \mathrm{~dB}$, a $10-\mathrm{dB}$ decrease of sensitivity level is necessary. For a lower absolute value of $\bar{G}_{p}$, a greater decrease of sensitivity level is required. The values of $\bar{G}_{p}$, which are smaller than $-1.5 \mathrm{~dB}$, permit a minor increase of sensitivity level.

For the case with a jamming signal at the pilot signal frequency and for $0 \leq a<1$ (where $a=A_{\text {int }} / A, A_{\text {int }}$-is amplitude of the jamming signal), all the above-stated results remain true. For $a>1$, the array will be focused in the direction of the jamming signal and it is necessary to add the reduction in directivity owing to main lobe shift from the direction of pilot signal arrival to the reduction in directivity due to cutting off channels. For evaluation of the directivity reduction in this case, it is necessary to substitute $\bar{a}=1 / a$ instead of $a$ and $\bar{\beta}=A_{\text {int }} / A_{\min }$ instead of $\beta$ in the abovestated expressions. The directivity reduction in this case will be determined in the direction of jamming signal arrival. For determination of the directivity reduction in the direction of 


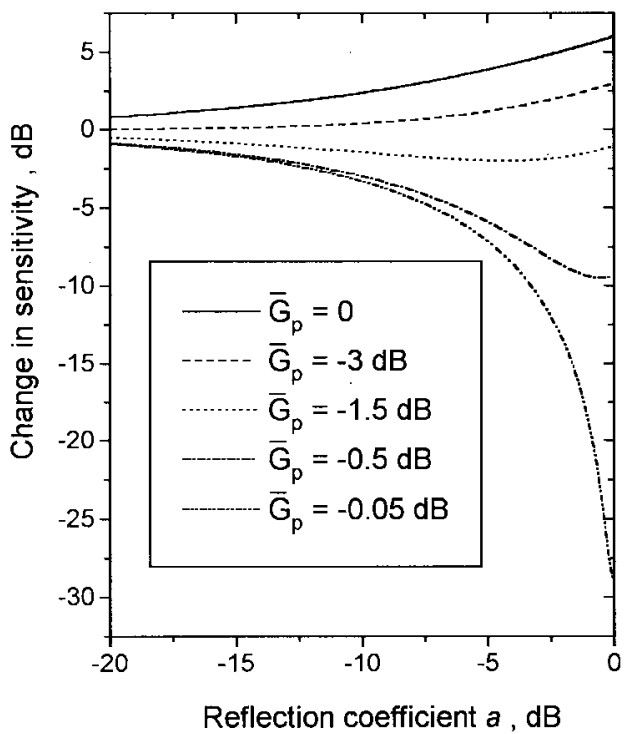

Fig. 4. Required change in sensitivity against reflection coefficient (17).

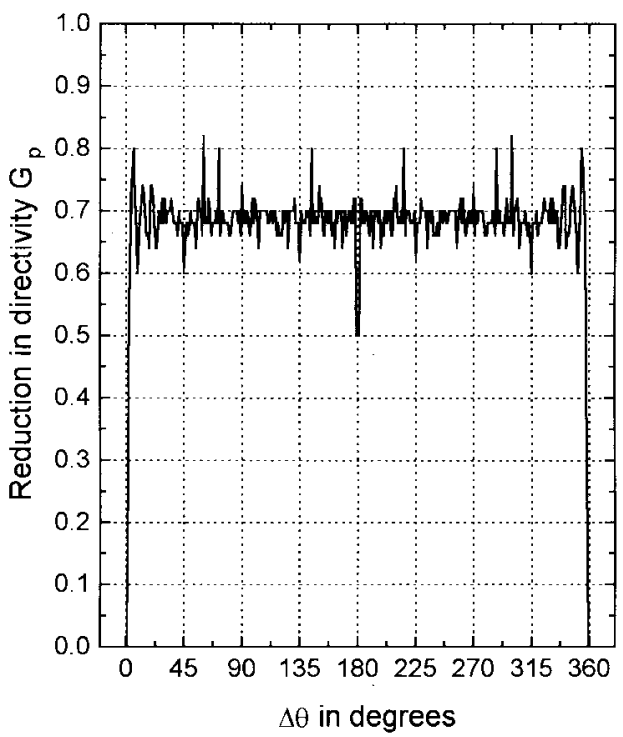

Fig. 5. Reduction in directivity against $\Delta \theta ; N=50, \beta=1.1, a=1$, $\theta_{1}=180^{\circ}$.

pilot signal arrival, it is necessary to take into account the difference of array directivity in the pilot and jamming signal directions.

Statistical description of self-phased array directivity and the results given above are of an average character and are true when the number of array elements exceeds ten. In a number of specific cases, a considerably greater reduction in directivity (presence of "ejections") can be observed. Let us consider a linear equally spaced self-phased array as an example. The structure of this array is shown in Fig. 1. A phase shift of direct and reflected pilot signal in $i$ th array element $\theta_{i}$ can be written as follows:

$$
\begin{aligned}
& \theta_{i}=\theta_{1}+\Delta \theta(i-1) \\
& \theta_{1}=\frac{2 \pi \Delta r}{\lambda_{p}}+\bar{\theta}, \quad \Delta \theta=\frac{2 \pi d}{\lambda_{p}}\left(\sin \left(\alpha_{2}\right)-\sin \left(\alpha_{1}\right)\right)
\end{aligned}
$$

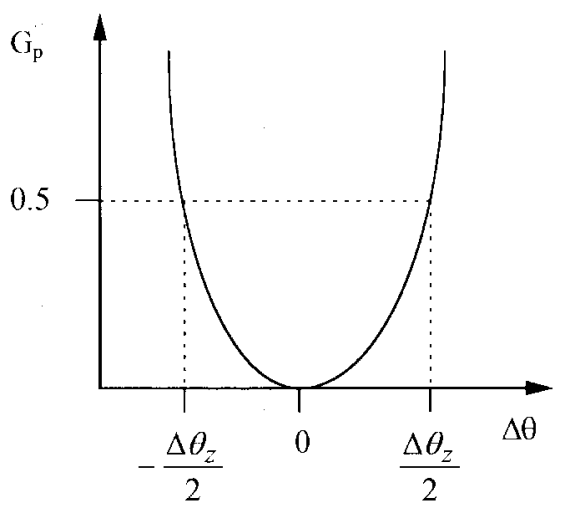

Fig. 6. Determination of the minimum zone width in the point $\Delta \theta=0$.

where $\theta_{1}$ is phase shift in the first element, $\Delta \theta$ is difference of phase shift from element to element, $\bar{\theta}$ is phase shift during reflection, $\lambda_{p}$ is wavelength of the pilot signal, $\Delta r$ is difference of path of the direct ray and reflected ray, $\alpha_{1}$ and $\alpha_{2}$ are direction of arrival angles of the direct and reflected signals accordingly, and $d$ is interelement distance. Using (2), (3) and (19), we can determine the number of cutoff channels and reduction in directivity $N_{d}=N_{d}\left(\theta_{1}, \Delta \theta, a, \beta\right)$ and $G_{p}=$ $G_{p}\left(\theta_{1}, \Delta \theta, a, \beta\right)$. Dependence of $G_{p}$ on $\Delta \theta$ for $a=1$ and $\beta=1.1$ is shown in Fig. 5. As it can be seen from this figure, the average value of $G_{p}$ is rather well described by (13). Individual maxima and minima are explained by combining of direct and reflected signals in phase or in opposite phase correspondingly in significant part of the array elements. The width of a minimum zone can be estimated by means of expressions (9) and (19). Half the array channels are in cutoff mode at the $-3-\mathrm{dB}$ boundary of the minimum zone in the point $\Delta \theta=0$ for $\theta_{1}=\pi\left(N_{d}=0.5 \mathrm{~N}\right.$, see Fig. 6); substituting first equality from (19) for $i=N / 2$ in (9) and using the equation $\pi$-arccos $(x)=\arccos (-x)$, one obtains

$$
\Delta \theta_{z} \approx \frac{4}{N} \arccos \left(\frac{\beta^{2}\left(1+a^{2}\right)-1}{2 \cdot a \cdot \beta^{2}}\right)
$$

where $\Delta \theta_{z}$-is the width of the minimum zone. The width of the other minimum and maximum zones has approximately the same value.

As (3) and (19) show, the worst case is realized for $\theta_{1}=\pi$ and $\Delta \theta=0\left(\alpha_{1}=\alpha_{2}\right)$, then $N_{d}=N$ and $G_{p}=0$. We should note that essential influence on $G_{p}$ is rendered by geometric parameters of the array. For instance, for $d / \lambda_{p}=0.5$ and $\theta_{1}=\pi, G_{p}=0$ for $\alpha_{1}=\alpha_{2}$ (one interference zone) and $\alpha_{1}=$ $\pi / 2, \alpha_{2}=-\pi / 2$ (and vice versa) - two other interference zones, i.e., in this case there are three interference zones. For $d / \lambda_{p}=1$ and $\theta_{1}=\pi, G_{p}=0$ for $\alpha_{1}=\alpha_{2}$-defines the first interference zone, $\alpha_{2}=\arcsin \left(1+\sin \alpha_{1}\right)$-defines the second interference zone, $\alpha_{2}=\arcsin \left(1-\sin \alpha_{1}\right)$-defines the third interference zone, and $\alpha_{1}=\pi / 2, \alpha_{2}=-\pi / 2$ (and vice versa) - defines the fourth and fifth interference zones. Further increase in $d / \lambda_{p}$ will lead to further increase of the number of these zones and, accordingly, to increase of probability of essential reduction in the array directivity (or to significant reduction in average directivity). This situation is similar to the emerging of additional grating lobes in the 
usual array with increasing interelement distance [3] or the emerging of additional intermodulation lobes in active arrays with increasing intermodulation order [4]-[7].

The magnitude $\Delta \theta$ varies in the interval $\left[-\Delta \theta_{\max }, \Delta \theta_{\max }\right]$ where $\Delta \theta_{\max }-$ maximum value $\Delta \theta$

$$
\Delta \theta_{\max }=\frac{4 \pi d}{\lambda_{p}}
$$

For the sake of simplicity, we assume that $\Delta \theta$ is distributed uniformly in this interval (the case of nonuniform distribution can be considered in the similar manner with the use of more complicated calculations). Then the probability of the essential reduction in directivity (i.e., falling within the interference zone) can be determined as follows:

$$
P_{z}=\frac{n_{z} \cdot \Delta \theta_{z}}{2 \cdot \Delta \theta_{\max }}
$$

where $n_{z}$-is the number of interference zones. This magnitude can be found using (19). We should note that (22) remains true when $n_{z} \Delta \theta_{z} \leq 2 \Delta \theta_{\max }$. If $\Delta \theta_{z}>2 \Delta \theta_{\max }$ then it is necessary to put $P_{z}=1$. Further, we shall consider the practically important case $\theta_{1}=\pi$ (this case is realized, for example, when $\Delta r \ll \lambda_{p}$ and $\bar{\theta}=\pi$ ). Then the condition for falling within the interference zone takes the form

$$
\Delta \theta=\frac{2 \pi d}{\lambda_{p}}\left(\sin \left(\alpha_{2}\right)-\sin \left(\alpha_{1}\right)\right)=2 \pi k, \quad k \text {-integer }
$$

and the number of these zones is determined as the number of values of $k$ for which this condition is fulfilled. Taking into account the symmetry of condition (23) with respect to $\alpha_{1}$ and $\alpha_{2}$, we obtain

$$
n_{z}=2 k_{\max }+1
$$

where $k_{\max }$-the maximum value of $k$ for which condition (23) is fulfilled. Since the maximum value of expression in brackets in (23) is equal to two, the expression for $k_{\max }$ takes the form

$$
k_{\max }=\left[\frac{2 d}{\lambda_{p}}\right]
$$

where $\left[{ }^{*}\right]$ means the integral part. Using (20), (22), and (25), we obtain

$$
P_{z} \approx \frac{\left(2 \cdot\left[\frac{2 d}{\lambda_{p}}\right]+1\right) \cdot \lambda_{p}}{2 \pi \cdot N \cdot d} \arccos \left(\frac{\beta^{2}\left(1+a^{2}\right)-1}{2 \cdot a \cdot \beta^{2}}\right) .
$$

As this expression shows, the probability of falling within an interference zone is determined by parameters of the array and a reflection coefficient. An increase of the number of the channels $(N)$ leads to decrease of this probability. It is explained by a decrease of interference zone width in accordance with (20). For two limiting cases of large and small ratio $d / \lambda_{p}$, the last expression is simplified and takes the form

$$
\begin{aligned}
& P_{z} \approx \frac{2}{\pi \cdot N} \arccos \left(\frac{\beta^{2}\left(1+a^{2}\right)-1}{2 \cdot a \cdot \beta^{2}}\right), \quad \frac{d}{\lambda_{p}}>1 \\
& P_{z} \approx \frac{\lambda_{p}}{2 \pi \cdot N \cdot d} \arccos \left(\frac{\beta^{2}\left(1+a^{2}\right)-1}{2 \cdot a \cdot \beta^{2}}\right), \quad \frac{d}{\lambda_{p}}<\frac{1}{2} .
\end{aligned}
$$

For large ratio $d / \lambda_{p}$, the probability $P_{z}$ does not depend on this magnitude, which is explained by a simultaneous increase in the number of interference zones $n_{z}$ and interval of $\Delta \theta$ change with increasing of $d / \lambda_{p}$. For small ratio $d / \lambda_{p}$, the probability $P_{z}$ will increase with decreasing of $d / \lambda_{p}$, which is explained by decrease of the interval of $\Delta \theta$ change and constant number of interference zones $\left(n_{z}=1\right)$ and its width. It should be noted that (20) and (26)-(28), as well as (9), remain true for $\beta \in\left[\beta_{m 2}, \beta_{m 1}\right]$. If $\beta<\beta_{m 2}$, it is necessary to put $P_{z}=1$. If $\beta>\beta_{m 1}$, it is necessary to put $P_{z}=0$. It is notable that the following connection exists between $P_{z}$ for large ratio $d / \lambda_{p}$ (27) and $\bar{G}_{p}$ (13)

$$
P_{z}=\frac{2\left(1-\bar{G}_{p}\right)}{N}
$$

i.e., increasing probability of falling within an interference zone will cause directivity to decrease.

Thus, increasing interelement distance leads to reduction in the array directivity not only due to emerging of grating lobes in the array pattern, but also due to an increase in the number of cutoff channels (influence by way of not only the primary signal channel, but also the pilot signal channel). However, this influence manifests itself in the case of a weak signal or a large reflection coefficient only.

\section{Self-PhASED ARRAY DiRECTIVITY FOR SEVERAL REFLECTED SIGNALS}

The results mentioned above can be generalized for the case of several reflected signals by means of the concept of the total reflected signal. The amplitude of the total reflected signal, which is equal to the sum of all reflected signals can be written in the following form:

$$
A_{\mathrm{sr}}=A \cdot \sqrt{\left(\sum_{i=1}^{m} a_{i} \cos \theta_{i}\right)^{2}+\left(\sum_{i=1}^{m} a_{i} \sin \theta_{i}\right)^{2}}
$$

where $a_{i}$ is the reflection coefficient for $i$ th reflected signal, $\theta_{i}$ is phase shift of direct and $i$ th reflected signal, $m$ is the number of reflected signals. The phase shift of the total reflected signal and the direct signal will look like

$$
\theta_{\mathrm{sr}}=\operatorname{arctg}\left(\frac{\sum_{i=1}^{m} a_{i} \sin \theta_{i}}{\sum_{i=1}^{m} a_{i} \cos \theta_{i}}\right)
$$

For this case, the equations for threshold values $\beta_{m 1}$ and $\beta_{m 2}$ take the form

$$
\beta_{m 1}=\frac{1}{1-\sum_{i=1}^{m} a_{i}}, \quad \sum_{i=1}^{m} a_{i}<1 ; \quad \beta_{m 2}=\frac{1}{1+\sum_{i=1}^{m} a_{i}} .
$$

Further, it is necessary to substitute the quantities $A_{\mathrm{sr}}$ and $\theta_{\mathrm{sr}}$ instead of $A_{\text {total }}$ and $\theta$, accordingly, in the expressions obtained above. In doing so, a rough estimate of average reduction in directivity can be obtained on the basis of (13) for the equivalent reflection coefficient $a=A_{\mathrm{sr}} / A$ on the assumption that different reflected signals are noncorrelated $\left(a_{e 1}^{\prime}\right)$ or are in phase $\left(a_{e 2}^{\prime}\right)$

$$
a_{e 1}=\sqrt{\sum_{i=1}^{m} a_{i}^{2}}, \quad a_{e 2}=\sum_{i=1}^{m} a_{i}
$$


A closer approximation of the average reduction in directivity can be obtained on the basis of (12) and (13) by determining the probability of cutting off channels with the use of (30) and the Monte Carlo method.

The use of the concept of the total reflected signal with parameters determined by (30) and (31) allows one to estimate the influence of several reflections on the directivity reduction by way of the primary signal channel, i.e., to calculate the magnitude of $G_{s}$, too.

Presence of a nonlinear element (mixer) in the structure of channel creates intermodulation interference. Interference impact on a self-phased array operation can be estimated by application of methods described in [4]-[7].

\section{Directivity of Self-Phased ARRAY FOR NONTHRESHOLD AMPLITUDE Characteristics of the Pilot Signal Channel}

An assumption was made above that the amplitude characteristic of the pilot signal channel is of the threshold type. However, in a number of cases this characteristic can essentially differ from that of the threshold type. In this situation, the dependence of the array directivity on the type of the amplitude characteristic of the pilot signal channel is of interest. This dependence can be established by appropriate modification of (2) on the basis of the following reasons. The amplitude of the primary signal at the output of a channel is determined by both the primary and pilot signal amplitudes at its input. Let us define the amplitude characteristic of the pilot signal channel $K\left(A_{\text {total }}\right)$ as

$$
K\left(A_{\text {total }}\right)=\frac{A_{w}}{A_{w 0}}
$$

where $A_{w}$ is amplitude of the total primary signal at the channel output when amplitude of the primary signal at the channel input is constant, $A_{w 0}$ is amplitude of the total primary signal at the channel output for rather large values of $A_{\text {total }}$ (in the proper operation mode when $K\left(A_{\text {total }}\right)=1$ ), and $A_{\text {total }}$ is amplitude of the total pilot signal at the channel input. Since the pilot signal is subjected to a limiting process prior to the mixer, the amplitude characteristic of the pilot signal channel will be largely determined by the amplitude characteristic of the limiter and will not depend on the primary signal amplitude (it is always possible to introduce such a dependence if necessary), i.e., $K\left(A_{\text {total }}\right)$ actually represents the normalized amplitude characteristic of the limiter.

The expression for the array directivity in this case takes the form

$$
\begin{aligned}
G= & \frac{A_{w, \text { out }}}{A_{1}} \\
= & \mid \sum_{i=1}^{N} \sqrt{1+a^{2}+2 \cdot a \cdot \cos \theta_{w i}} \cdot K\left(A_{\text {total }, i}\right) \\
& \cdot \exp \left(j\left(\psi_{w i}-\psi_{p i}\right)\right) \mid
\end{aligned}
$$

where $A_{w, \text { out }}$ is amplitude of the primary signal at the array output, $A_{1}$ is amplitude of the primary signal at the channel output in the absence of reflection, $\theta_{w i}$ phase shift of the direct and reflected primary signal at the input of $i$ th channel, $A_{\text {total }, i}$ is magnitude $A_{\text {total }}$ at the input of $i$ th channel, and $\psi_{w i}$ and $\psi_{p i}$ are phases of the total primary and pilot signals at the input of $i$ th channel, accordingly. The expressions for magnitudes $\psi_{w i}$ and $\psi_{p i}$ can be found in [2]. Thus, it is not obviously possible to present array directivity in the form of (1), i.e., to separate the influence of the reflections into influence by way of the primary signal channel and influence by way of the pilot signal channel in this case. The possibility of representation of array directivity in the form of (1) is substantiated only by the threshold type of amplitude characteristic of the pilot signal channel, when $K\left(A_{\text {total }}\right)$ takes only two values-zero and one.

\section{CONCLUSION}

The analysis of self-phased arrays under conditions of multipath propagation has shown that the pilot signal channel can significantly influence the array directivity. It is worthwhile using the average reduction in the array directivity at the expense of cutting off some channels as a measure of this influence. The greatest reduction in the array directivity is observed for the case of small amplitudes of the pilot signal or for a large reflection coefficient. If the amplitude of the pilot signal exceeds the sensitivity level, the average reduction in directivity does not exceed $3 \mathrm{~dB}$. A significant influence on the directivity reduction is rendered by geometric parameters of the array (interelement distance), e.g., increase of the interelement distance leads to increase of the number of interference zones. The equations given in the paper permit the calculation of the required change of sensitivity of the pilot signal channel for the given average reduction in directivity and reflection coefficient.

The probability of the cutting off a channel [the expression (11)] can be used as an alternate measure of influence of the pilot signal on array directivity. In this case, the required change of sensitivity is determined for the given reflection coefficient and the probability of cutting off a channel. The separation of influence of reflections on self-phased array directivity into influence by way of the primary signal channel and influence by way of the pilot signal channel is possible in the case of the threshold amplitude characteristic of the pilot signal channel only. In other cases such separation is not possible.

The suggested method allows one to determine an average reduction in directivity of self-phased array for the case of several reflected signals. The rough estimate of this magnitude can be obtained on the basis of the equivalent reflection coefficient on the assumption that different reflections are noncorrelated or are in phase. A closer approximation requires statistical simulation with the use of the Monte Carlo method.

The obtained results can be used for analysis and design of self-phased arrays in complicated electromagnetic environment, e.g., in the presence of reflections (multipath propagation) or jamming signals at the frequency of the pilot signal. 


\section{REFERENCES}

[1] P. V. Brennan, "An experimental and theoretical study of self-phased arrays in mobile satellite communications," IEEE Trans. Antennas Propagat., vol. 37, pp. 1370-1376, Nov. 1989.

[2] S. L. Loyka, "Directivity of self-phased arrays in conditions of multipath propagation," in Proc. 7th Int. Conf. HF Radio Syst. Tech., Nottingham, U.K., July 1997.

[3] D. M. Sazonov, Microwave Circuits and Antennas. Moscow, Russia: Mir, 1990.

[4] _ "Characteristics of receiving intermodulation channel of active array antennas," Int. J. Electron., vol. 80, pp. 595-602, Apr. 1996.

[5] __ "Interference immunity of active array antennas in rigid electromagnetic environment," in Proc. ÕI Int. Microwave Conf. XI "MIKON96", Warsaw, Poland, May 1996, vol. 1, pp. 57-61.

[6] _ "Intermodulation receiving channels in active array antennas," Izvestia Vuzov. Radioelectron. (Radioelectron. Commun. Syst.), vol. 39, pp. 68-74, Feb. 1996 (in Russian).

[7] _ "Conditions of existence of main lobes in two-signal spatial selectivity characteristic of active array antenna," Izvestia Vuzov. Raioelectron. (Radioelectron. Commun. Syst.), vol. 39, pp. 3-9, June 1996 (in Russian).

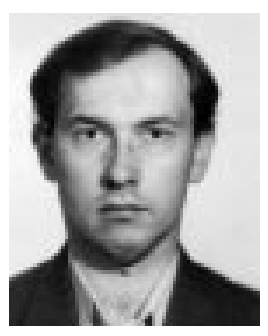

Sergey L. Loyka (M'97) was born in Minsk, Republic of Belarus, on August 6, 1969. He received the M.S. (honors) degree from Minsk Radioengineering Institute, Minsk, Republic of Belarus, in 1992, and the Ph.D. degree in electrical engineering from the Belorussian State University of Informatics and Radioelectronics, Minsk, Republic of Belarus, in 1995

Since 1995, he has been Senior Researcher of the Electromagnetic Compatibility Laboratory, Belorussian State University of Informatics and Radioelectronics, Minsk, Republic of Belarus. He has over 30 publications in the area of electromagnetic compatibility, active and self-phased antenna arrays, nonlinear circuit analysis, and computer-aided modeling and design.

Dr. Loyka is a member of the New York Academy of Sciences. 